\title{
The Role of Pura in Neuronal Development, the Progress in the Current Researches
}

\author{
Chengmin Yuan ${ }^{1}$, Ping Li ${ }^{2}$, Shanshan Guo ${ }^{1}$, Bingying Zhang ${ }^{2}$, Tao Sun ${ }^{1}$ and Jianqi Cui ${ }^{1,2}$
}

${ }^{1}$ Ningxia Key Laboratory of Cerebrocranial Diseases, Incubation Base of National Key Laboratory, Ningxia Medical University, Yinchuan, Ningxia, PR China

${ }^{2}$ Department of Biochemistry and Molecular Biology, School of Basic Medical Sciences, Ningxia Medical University, Yinchuan, Ningxia, PR China

Corresponding author: Dr. Jianqi Cui, Ningxia Key Laboratory of Cerebrocranial Diseases, Incubation Base of National Kay Laboratory, Ningxia Medical University, 1160 Shengli Street, Yinchuan, Ningxia, 750004, PR China, Tel: 15296900235; Fax: 86-951-6880697; E-mail: Jianqi@gmail.com

Received: Sep 26, 2016; Accepted: Oct 25, 2016; Published: Oct 31, 2016

Citation: Yuan C, Li P, Guo S, et al. The Role of Pura in Neuronal Development, the Progress in the Current Researches. J Neurol Neurosci. 2016, 7:5.

\section{Abstract}

As a ubiquitous protein in the body, Pura has been considered to possess multiple regulatory functions from DNA replication and transcription to RNA transport and translation. The recent studies have proved that Pura protein played a vital role in neuronal development with the solid and abundant evidence both in transgenic mice and human genetic DNA analysis. The Pura knocked out mice models have been successfully established in two independent research groups and their results demonstrated that lack of Pur $\alpha$ alters postnatal brain development. Mice lacking of Pura display decreased neurogenesis and impaired neuronal development. In the mouse brain, the expression level of Pura seems to be regulated with brain development. It starts to express Pura after birth and quickly reach to highest level in the third week. The recent studies also have proved that some neurodegenerative disease also linked with Pura, such as fragile $\mathrm{X}$-associated tremor/ataxia syndrome. rCGG-mediated neuronal toxicity could be modulated by heterogeneous nuclear ribonucleoprotein A2/B1 and Pura, which is rCGG-repeat-binding proteins. The $5 q 31.3$ Microdeletion Syndrome is another disorder that is associated with the mutation of Pura gene, it has been demonstrated that all the symptoms such as the neurocognitive impairment, neurodevelopmental delay and learning disability are caused by the mutation of Pura gene. In this mini review, we will retrospectively review the current progress of Pura in neuronal development and try to figure out the connections between these observed phenomena and the biological function of Pura.

Keywords: Pura; Neuronal development; Gene mutation; Knock out; Seizures

\section{Introduction}

Pura [1], encoded by PURA gene, could bind to singlestranded DNA and RNA [2,3] in a sequence-specific way. Pura is a highly conserved protein throughout the whole evolution; it has been reported that between mouse and human beings, there are only 2 amino acid residues differences in the sequence of all the 322 amino acids of Pura [3]. Pur $\alpha$ has a broad potential functions, the increasing research works have demonstrated that the initiation of DNA replication, gene transcription, and the compartmentalized mRNA translation [4-6]. In addition, Pura is also involved in cell cycle regulation, oncogenic transformations [1,7-9], and DNA repair [10,11]. Pura is though expressed in every metazoan tissue [5], it predominantly plays a regulatory function in the developmental stage $[12,13]$ in a tissue-specific way. Although the precise roles of Pur $\alpha$ in the brain are still debatable, most of the current research works demonstrated that Pura possibly participates both in neuronal proliferation and in the maturation of dendrites. In Pura knock out mice, the animals look normal at birth, but soon develop neurological deficit at 2 weeks of age, and die within 4 weeks [14]. Another recently discovered neurodegenerative disorder is fragile $\mathrm{X}$-associated tremor/ataxia syndrome [15], the adult carriers of premutation alleles (55 to 200 CGG repeats) of the fragile $X$ mental retardation $1[16]$ gene $[17,18]$ would be affected. In addition, another neuronal developmental disorder linked with Pura is 5q31.3 Microdeletion Syndrome reported by Lalani et al. [19] and Hunt et al. [20]. For more detailed information about Pura please refer to the review written by Johnson [5,21], White [22] and Chai et al. [23]. In this review, we will mainly discuss the functions of Pura protein on the neuronal development, the transgenic model of Pura as well as the functions of Pura on neurological disorders.

\section{Overview of Pur $\alpha$ and its functions on neuronal development}

Pura is a ubiquitous nucleic acid-binding protein originally purified from mouse brain. Pura has an ability to bind to a 
DNA sequence derived from the promoter of the mouse myelin basic protein gene $[12,24]$. Human Pura possesses an ability to bind to a DNA sequence presented upstream of the human c-Myc gene. The Pura cDNA was cloned from HeLa cells and its sequence was also analyzed [3]. Pura has a very high conservation in their sequence and there is only 2 out of 322 amino acid residues different between the mouse [25] and human beings [2]. Same as other transcriptional factors, Pura also has a modular structure with a central-binding domain containing 3 class $I$ repeats and 2 class 2 repeats. There is a glycine-rich domain in its N-terminal and a "psycho" domain and glutamine-rich domain and glutamate-rich domain in its Cterminal. Its DNA binding domain is intensely conserved throughout evolution [22] (Figure 1).

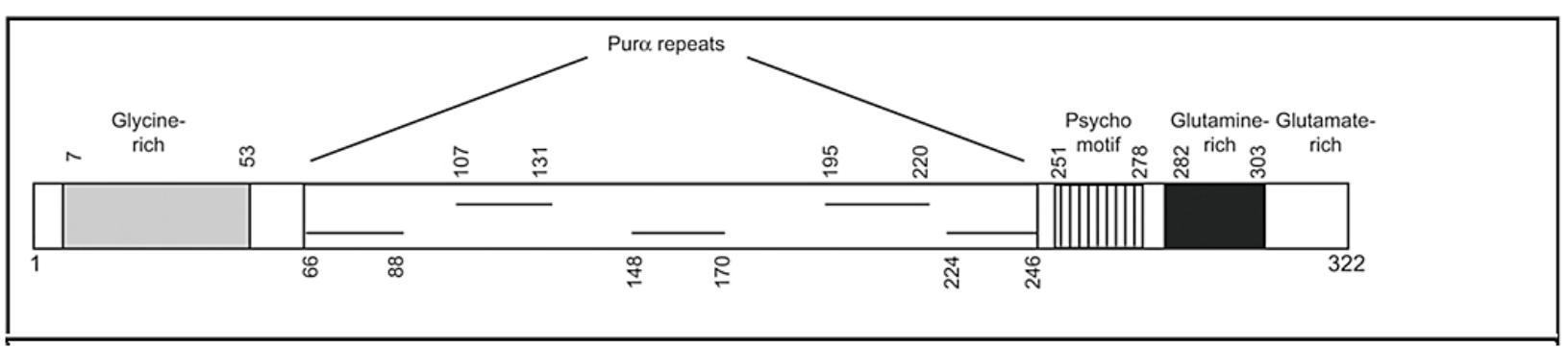

Figure 1 The structure of Pura protein.

Pur family includes three different proteins, Pura, Pur $\beta$ [2] and Pury. Since they use the alternative polyadenylation sites [26], there are two isoforms existed, and in almost every metazoan tissue can detect the expression of Pur proteins [5]. As a multifunctional protein, Pura [5] can bind to both DNA and RNA and because of this property, it has been reported that the initiation of DNA replication, control of transcription and mRNA translation $[2,4]$ are all associated with Pura. It has been reported that the DNA sequences close to viral and cellular origins of replication could be associates with Pur $\alpha$, Pura might have an ability to destabilize DNA helix [27], as we know that the initiation of transcription and replication requires unwinding of duplex DNA, the critical role of Pura in neuronal development and synaptogenesis is undoubted. Being a multifunctional protein, Pur $\alpha$ binds to single-stranded nucleic acids in a sequence-specific way to fulfill its regulatory functions in many critical life activities, which could be the reason why its structure is strongly conserved through the evolution [5]. The extensive characteristics of Pura is its ability to bind to single-stranded DNA and RNA in a sequence specific way, therefore, it has been originally cloned from HeLa cell according to its affinity for a single-stranded DNA element with the sequence GGNGGN [2]. Since Pura plays critical roles in DNA replication and gene transcription [4,5], the increasing research works reported that it also acted as a critical component in the compartmentalized translation of RNA [6]. In addition, as a comprehensively expressed protein, Pura plays developmental and tissue-specific regulatory roles in the central nervous system $[14,24]$. Khalili et al. reported that during embryogenesis period Pura protein levels kept in a quite low level in mouse brain, but after the first postnatal week, it quickly begin to increase and reach their peak at 18 days postnatal. From then on, Pura is retained at comparatively high levels in the adult brain [14]. Consequently, these data suggest that Pura levels inversely correlate during mouse brain development.

The distribution of Pura in neurons is specific and it has been reported that Pura is mainly expressed in the cytoplasm, particularly in dendrites at branch points [6]. Interestingly, Pura is also to be found in a complex form with polyribosomes and heterogeneous nuclear ribonucleoproteins (hnRNP) proteins existed in the neuron. Pura knockout mice die shortly after birth with a variety of organ defects [14]. In particular, mice lacking Pura show neurological dysfunction, and histopathological examination of knockout mouse brains has shown defects in neuronal cell development and differentiation which leads to neuronal dropout as well as abnormal and delayed corticogenesis. Defects in dendrite development throughout the cortex, the hippocampus, and the cerebellum were observed $[14,28]$. Hippocampal neurons fail to form synaptic connections in the absence of Pura, as visualized by a significant lack of post-synaptic density-95 (Psd95) foci. Similar, a possible role in regulation of immune cell function and response to hypoxia has also been attributed to Pura [28].

\section{Postnatal brain development was affected when Pura has been disrupted in knock out mice model}

Previous experiments have demonstrated that Pura is essential for postnatal development and developmentally coupled cellular proliferation in the mouse. Khalili et al. [14] has reported that when both alleles of PURA gene have been disrupted, the mice displayed special features. The mice seem normal at birth, but gradually developed neurological problems at 2 weeks of age, also companied with some abnormal signs such as severe tremor and spontaneous seizures. The mice were bound to die by 4 weeks.

Histological examination demonstrated that the numbers of neurons in regions of the hippocampus and cerebellum in PURA knock-out mice was much less than that in the agematched wild type littermates, and also, lamination of these regions is deviant at time of death. MCF7 is a protein marker for DNA replication, the PURA knock-out mice demonstrated that the proliferation of precursor cells is deficient in these 
regions when MCF7 levels were immunohistochemically analyzed. These phenomenon were also observed in several other tissues, such as those of myeloid linage, but in heterozygous mice, these aberrant phenomenon is relatively less severe than that in homozygous mice. Histological examination also demonstrated that the level of myelin and glial fibrillary acidic protein were reduced both in oligodendrocytes and astrocytes, which implies that these cells undergo a pathological development. A crucial time for cerebellar development appears at the 5th day after birth, as a signal protein marker for these is the Pura and Cdk5, the expression level of them were both in their highest levels in bodies and dendrites of Purkinje cells of wild type mice, in normal condition, Pura and Cdk5 can be coimmunoprecipitated from brain lysates of wild type mice, but unfortunately, both of them were missing in dendrites of PURA knock-out mice. In accompany with these observation, the level of both phosphorylated and non-phosphorylated neurofilaments in dendrites of the Purkinje cell layer and of synapse formation in the hippocampus were all remarkably reduced with immunohistochemical studies. All above discoveries indicate that Pura participates in the developmentally timed DNA replication in specific cell types, this might be a new arising role of Pur $\alpha$ in compartmentalized RNA transport and translation in neuronal dendrites [14].

Interestingly, another comparable work reported by Hokkanen et al. reported that deficiency of Pura changes postnatal brain development and gives rise to megaloencephaly [29]. In their generated Pura-deficiency mice model, they found that the mice were born with no obvious pathological condition and could be raised to 6 months but not died at week 4 as reported by Khalili's work. Hokkanen thought that the postnatal proliferation of neuronal precursor cells both in the hippocampus and in the cerebellum were only extended when Pura was disrupted, but the overall number of post mitotic neurons were not affected. In previous studies, the transition of dendritic protein MAP2 is thought to be Puradependent, Hokkanen's work also found the changes of this protein's expression and distribution. Same as Khalili's work, they also found that the PURA knock-out mice developed a continuous tremor at the age of 2 weeks and this symptom continued throughout lifetime; this observation is consistent with Khalili's report, it indicates that disruption the endogenous level of Pura expression would be associated with the onset of epilepsy, this phenomenon is also confirmed by other researches related to the changes of Pura $[19,20]$, about this we will discuss later in the review.

In contrast to Khalili's work, some other new findings such as a megaloencephaly, axonal swellings and hyper phosphorylation of neurofilaments were observed in PURA knock-out mice. Nevertheless, both of these two research works confirmed the effects of Pura on the brain development, but there are still some differences on the observed phenotypes, especially whether the Pura knock-out mice die at the 4th week after the birth or can live to the adulthood, the reason for this is not very clear right now. Hokkanen's work did not show the western blotting results of the of expression level of Pura in their knock out mice, the efficiency of knock out of Pura is complete or not, or it might be only a truncated form of Pura being knocked out, all of these has to be considered as a reason for this differences.

\section{PURA mutation is associated with $5 q 31.3$ Microdeletion Syndrome}

$5 q 31.3$ Microdeletion Syndrome is a rare disorder and firstly reported by Shimojima et al. [30], which is characterized by severe developmental delays, distinctive facial features, and delayed myelination. The subsequent researches continually reported this new discovered disorder associated with neuronal abnormalities, including neonatal hypotonia, encephalopathy, and severe developmental delay, with or without seizure. All the researches demonstrated that the disorder is linked to the minimal critical deletion interval harbors three genes [30-33].

Recently, Lalani et al. has reported 11 individuals with clinical features of $5 q 31.3$ Microdeletion Syndrome and they found de novo mutations in PURA within the critical region [19]. And their data demonstrated that the severe neurological abnormalities observed in this syndrome is caused by the mutation of PURA gene. Among 2908 successive subjects, 11 individuals were found to have de novo heterozygous mutations in the single-exon gene PURA, in which there were four truncating (three nonsense and one frameshift), five missense and two in-frame deletions. All 11 individuals appeared significant hypotonia at birth, other symptoms included respiratory insufficiency (central and obstructive sleep apnea and recurrent pulmonary aspiration). Since the patients often encountered early-onset feeding difficulties with moderate dysphagia and evidence of tracheal aspiration, nasogastric or gastric-tube feeding were often required. Another common features observed in the subjects with $5 q 31.3$ Microdeletion Syndrome is their myopathic facies, which were characterized as an open mouth and high arched palate. There were 4 out of 11 individuals presented with hypomyelination or myelin-maturation delay when checked by brain-imaging studies. Another frequently observed symptoms in infancy were myoclonic jerks which caused 6 out of 11 children to progress to develop epilepsy. Most individuals had abnormal EEG and two individuals were diagnosed LennoxGastaut syndrome. It has to be pointed out that the exome sequencing is a valuable method for the diagnosis of this disorder and it is helpful distinguish this disease with other disorders. All the above mentioned results indicate that severe neurodevelopmental profile of $5 q 31.3$ Microdeletion Syndrome is closely associated with PURA mutations since all the individuals with this mutation share the common pattern of clinical manifestations.

A similar results was reported by Hunt et al. they found de novo mutations of PURA in family trios, which causes severe, sporadic, undiagnosed neurodevelopmental delay and learning disability through a whole exome sequencing [29]. There were four subjects has been identified with this de novo mutation which causes the structural changes of the PURA encoded protein Pura, usually it is considered as a transcriptional activator. The four mutations including two different 
frameshifts, one in-frame deletion and one missense mutation had been identified through a whole exome sequencing. Although the phenotype of the mutations in this gene is variable, the clinical manifestations are common to all individuals, which included moderate to severe neurodevelopmental delay and learning disability, neonatal hypotonia, early feeding difficulties and seizures, or 'seizurelike' movements. In addition to the above findings, this disorder might also be associated with anterior pituitary dysregulation. When Pura repeat III was disrupted, a more severe phenotype appeared, so it is reasonable to think there might be a possible genotype-phenotype correlation existed in the brain though psychomotor developmental outcomes seem variable among patients.

\section{Pura and Fragile $X$ Tremor/Ataxia Syndrome}

Fragile $\mathrm{X}$ syndrome is previously described by Mareni et al. [31] reported that individuals with the form of $X$-linked mental retardation is associated with the presence of a cytologically variant $X$ chromosome in which there is a secondary constriction or "fragile site" at Xq 27-28 (Fra X) existed. From then on, the increasingly research works focused on this field and confirmed that this disorder is caused primarily by expansion of the CGG trinucleotide repeat in the 50 untranslated region (50 UTR) of the fragile $X$ mental retardation 1 (FMR1) gene [34,35]. It has been demonstrated that fully affected individuals have more than 200 CGG repeats that are referred to as full mutation alleles, whereas the normal individuals generally only possess between 5 and 54 repeats. The FMR1 gene is considered as precursor of full mutation alleles when they expanded, they can change from a premutation alleles into full mutation form through genetic instability during maternal germline transmission [35]. The premutation carriers of fragile $\mathrm{X}$-associated tremor/ataxia syndrome (FXTAS), which is considered as a recently identified neurodegenerative disorder, were all found in male and female individual carriers [32,33]. Although the incidence to develop FXTAS is much higher in male carriers, female carriers also can develop FXTAS [33]. There is the ubiquitin-positive intranuclear inclusion present in both neurons and astrocytes throughout the brain and it has been considered as the remarkable neuropathological features of FXTAS [32] and the progressive intention tremor, gait ataxia, parkinsonism, autonomic dysfunction, and cognitive decline were comprised of the most common clinical manifestations of FXTAS [36]. Additionally, the marked dropout of Purkinje cells, Purkinje axonal torpedoes, and Bergmann gliosis were also observed in the cerebellum of FXTAS patients. Immunohistochemical examination demonstrated that there was no intranuclear inclusions present in Purkinje cells, and they appeared in the dentate nucleus in a small number of neurons and diffusely distributed in cerebellar astrocytes [37].

The research work from Jin et al. demonstrated the mechanism of Pura in the FXTAS syndrome, they successfully established a Drosophila model of fragile $X$ tremor/ataxia syndrome in which they found that Pura could bind to rGGG repeats and modulate repeat-mediated neurodegeneration [16]. They suggested that rCGG-repeat-binding proteins (RBPs) (in which Puro and hnRNP A2/B1 has been identified as RBPs) may be sequestered from their normal function by rCGG binding since transcribed premutation repeats alone are sufficient to cause neurodegeneration. They also show that Puro and rCGG repeats interact in a sequence-specific way and it is strongly conserved through evolution. rCGG-mediated neurodegeneration could be suppressed with overexpression of Pur $\alpha$ in Drosophila in a dose-dependent way. Additionally, it also has been illustrated that Pura existed in the inclusions of FXTAS patient brains. Above all, it is not difficult to conclude that Pur $\alpha$ plays an important role in the pathogenesis of FXTAS since rCGG repeat sequestration of specific RBPs and result in neuronal cell death.

Interestingly, the research work from another group supports Jin's work. Tan et al. [38] also found that in an FXTAS Drosophila model, gypsy, a specific retrotransposon, played an important role in the regulation of rCGG-mediated neurodegeneration when it was activated. On the other hand, the neuronal toxicity caused by rCGG repeats could be suppressed when gypsy was knockdown. They also found that hnRNP A2/B1, one of the rCGG-repeat-binding protein is involved in this process through interaction with heterochromatin protein 1 . All these findings indicate an important role for retrotransposition in neurodegeneration [38].

The role of Pura in rCGG-mediated neurodegeneration was further investigated by Qurashi et al. [39]. They identified more than 100 proteins that interact with Pura through a proteomic approach. It is interesting to notice that rCGGmediated neurodegeneration could be regulated by $\mathrm{Rm62}$, which is a kind of p68 RNA helicase existed in Drosophila. The expression of Rm62 could be reduced post-transcriptionally by rCGG repeats and result in the Hsp70 transcripts accumulated in the nucleus and, which will act as additional mRNA to participate in stress and immune response. All these data might point to a new mechanism of FXTAS pathogenesis in which, the nuclear exports were impaired by these mRNA abnormally accumulated inside the nucleus.

\section{Conclusions}

Pura is an important transcriptional factor in different cellular processes. Pur $\alpha$ plays a critical role in the developing and mature brain and targeted disruption of the PURA gene in both alleles has also confirmed its functions in postnatal brain development. Pura-deficient mice appear a serial pathologic phenotype in neuronal system manifested by severe tremor and spontaneous seizures. The neuronal cells were found very low in number in hippocampus and cerebellar regions in addition to many other abnormalities. Although other similar work demonstrated that the PURA-/- mouse can be raised to 6 months without dying, but the brain development was altered when Pura is knocked out. Both model displayed the phenomenon that disruption the Pura expression will lead the mouse to induce seizures. All these results indicate some connections between Pur $\alpha$ and epilepsy. 
Pura is also associated with $5 q 31.3$ Microdeletion Syndrome and the mutation of PURA would be the cause of this disorder which has been approved by different individual research groups. The patient with 5q31.3 Microdeletion Syndrome displayed severe neurological conditions, including neonatal hypotonia, feeding difficulties, an abnormal electroencephalogram(EEG), hypomyelination and severe psychomotor delay, the spontaneous seizure or tremor is also observed in the patient. With whole exome sequencing analysis, it has been confirmed that the mutation of Pura can cause this disorder.

Pura is also involved in the fragile $X$ tremor/ataxia syndrome since it can bind to rCGG repeats and modulate repeatmediated neurodegeneration. Overexpression of Pura in Drosophila could suppress rCGG-mediated neurodegeneration in a dose-dependent manner.

Above all, Pura is an important transcriptional factor and play a critical role in the neuronal development. Targeted disruption the expression of Pura would lead severe neurological conditions, especially, it might be involved in the onset of seizure.

\section{Acknowledgments}

This work was supported by grants from NFCS to Dr. Jianqi Cui (81260197) and National 973 Project to Dr. Jianguo Niu (2014BC560711) and Grant of Dominant Subject Group for Tao Sun (XY201511) supported by Ningxia Medical University scientific research project.

\section{References}

1. Barr SM, Johnson EM (2001) Ras-induced colony formation and anchorage-independent growth inhibited by elevated expression of Puralpha in NIH3T3 cells. J Cell Biochem 81: 621-638.

2. Bergemann AD, Johnson EM (1992) The HeLa Pur factor binds single-stranded DNA at a specific element conserved in gene flanking regions and origins of DNA replication. Mol Cell Biol 12 1257-1265.

3. Bergemann AD, Ma ZW, Johnson EM (1992) Sequence of cDNA comprising the human pur gene and sequence-specific singlestranded-DNA-binding properties of the encoded protein. Mol Cell Biol 12: 5673-5682.

4. Gallia GL, Johnson EM, Khalili K (2000) Puralpha: a multifunctional single-stranded DNA- and RNA-binding protein. Nucleic Acids Res 28: 3197-3205.

5. Johnson EM (2003) The Pur protein family: clues to function from recent studies on cancer and AIDS. Anticancer Res 23 2093-2100.

6. Johnson EM, Kinoshita $Y$, Weinreb DB, Wortman MJ, Simon R, et al. (2006) Role of Pur alpha in targeting mRNA to sites of translation in hippocampal neuronal dendrites. J Neurosci Res 83: 929-943.

7. Stacey DW, Hitomi M, Kanovsky M, Gan L, Johnson EM (1999) Cell cycle arrest and morphological alterations following microinjection of NIH3T3 cells with Pur alpha. Oncogene 18: 4254-4261.
8. Darbinian N, Gallia GL, King J, Del Valle L, Johnson EM, et al. (2001) Growth inhibition of glioblastoma cells by human Pur(alpha). J Cell Physiol 189: 334-340.

9. Lezon GK, Najfeld V, Johnson EM (2001) Deletions of PURA, at 5q31, and PURB, at 7p13, in myelodysplastic syndrome and progression to acute myelogenous leukemia. Leukemia 15: 954-962.

10. Wang $\mathrm{H}$, White $\mathrm{MK}$, Kaminski $\mathrm{R}$, Darbinian $\mathrm{N}$, Amini $\mathrm{S}$, et al. (2008) Role of Puralpha in the modulation of homologous recombination-directed DNA repair by HIV-1 Tat. Anticancer Res 28: 1441-1447.

11. Wang $H$, Wang $M$, Reiss $K$, Darbinian $S N$, Johnson $E M$, et al. (2007) Evidence for the involvement of Puralpha in response to DNA replication stress. Cancer Biol Ther 6: 596-602.

12. Haas S, Gordon J, Khalili K (1993) A developmentally regulated DNA-binding protein from mouse brain stimulates myelin basic protein gene expression. Mol Cell Biol 13: 3103-3112.

13. Tretiakova A, Gallia GL, Shcherbik N, Jameson B, Johnson EM, et al. (1998) Association of Puralpha with RNAs homologous to $7 \mathrm{SL}$ determines its binding ability to the myelin basic protein promoter DNA sequence. J Biol Chem 273: 22241-22247.

14. Khalili K, Del Valle L, Muralidharan V, Gault WJ, Darbinian N, et al. (2003) Puralpha is essential for postnatal brain development and developmentally coupled cellular proliferation as revealed by genetic inactivation in the mouse. Mol Cell Biol 23: 6857-6875.

15. Daigle JG, Krishnamurthy K, Ramesh N, Casci I, Monaghan J, et al. (2016) Pur-alpha regulates cytoplasmic stress granule dynamics and ameliorates FUS toxicity. Acta Neuropathol 131: 605-620.

16. Jin P, Duan R, Qurashi A, Qin Y, Tian D, et al. (2007) Pur alpha binds to rCGG repeats and modulates repeat-mediated neurodegeneration in a Drosophila model of fragile $\mathrm{X}$ tremor/ ataxia syndrome. Neuron 55: 556-564.

17. Hagerman RJ, Leehey M, Heinrichs $\mathrm{W}$, Tassone F, Wilson R, et al. (2001) Intention tremor, parkinsonism, and generalized brain atrophy in male carriers of fragile X. Neurology 57: 127-130.

18. Jacquemont S, Hagerman RJ, Leehey M, Grigsby J, Zhang L, et al. (2003) Fragile $X$ premutation tremor/ataxia syndrome: molecular, clinical, and neuroimaging correlates. Am J Hum Genet 72: 869-878.

19. Lalani SR, Zhang J, Schaaf CP, Brown CW, Magoulas P, et al. (2014) Mutations in PURA cause profound neonatal hypotonia, seizures, and encephalopathy in $5 q 31.3$ microdeletion syndrome. Am J Hum Genet 95: 579-583.

20. Hunt D, Leventer RJ, Simons C, Taft R, Swoboda KJ, et al. (2014) Whole exome sequencing in family trios reveals de novo mutations in PURA as a cause of severe neurodevelopmental delay and learning disability. J Med Genet 51: 806-813.

21. Johnson EM, Daniel DC, Gordon J (2013) The pur protein family: genetic and structural features in development and disease. J Cell Physiol 228: 930-937.

22. White MK, Johnson EM, Khalili K (2009) Multiple roles for Puralpha in cellular and viral regulation. Cell Cycle 8: 1-7.

23. Chai J, Li Y, Wang H, Cui J (2015) DNA Damage and Repair, Neurodegeneration and the Role of Pura in DNA Repair 7: 4:5.

24. Haas $S$, Thatikunta $P$, Steplewski A, Johnson EM, Khalili K, et al. (1995) A 39-kD DNA-binding protein from mouse brain 
stimulates transcription of myelin basic protein gene in oligodendrocytic cells. J Cell Biol 130: 1171-1179.

25. Ma ZW, Bergemann AD, Johnson EM (1994) Conservation in human and mouse Pur alpha of a motif common to several proteins involved in initiation of DNA replication. Gene 149: 311-314.

26. Liu H, Johnson EM (2002) Distinct proteins encoded by alternative transcripts of the PURG gene, located contrapodal to WRN on chromosome 8, determined by differential termination/polyadenylation. Nucleic Acids Res 30: 2417-2426.

27. Darbinian N, Gallia GL, Khalili K (2001) Helix-destabilizing properties of the human single-stranded DNA- and RNA-binding protein Puralpha. J Cell Biochem 80: 589-595.

28. Kong T, Scully M, Shelley CS, Colgan SP (2007) Identification of Pur alpha as a new hypoxia response factor responsible for coordinated induction of the beta 2 integrin family. J Immunol 179: 1934-1941.

29. Hokkanen S, Feldmann HM, Ding H, Jung CK, Bojarski L, et al. (2012) Lack of Pur-alpha alters postnatal brain development and causes megalencephaly. Hum Mol Genet 21: 473-484.

30. Shimojima K, Isidor B, Le Caignec C, Kondo A, Sakata S, et al. (2011) A new microdeletion syndrome of $5 q 31.3$ characterized by severe developmental delays, distinctive facial features, and delayed myelination. Am J Hum Genet Part A 155A: 732-736.

31. Mareni C, Migeon BR (1981) Fragile X syndrome: search for phenotypic manifestations at loci for hypoxanthine phosphoribosyltransferase and glucose-6-phosphate dehydrogenase. Am J Hum Genet 33: 752-761.

32. Hagerman PJ, Hagerman RJ (2004) Fragile X-associated tremor/ ataxia syndrome (FXTAS). Ment Retard Dev Disabil Res Rev 10: 25-30.
33. Hagerman RJ, Leavitt BR, Farzin F, Jacquemont S, Greco CM, et al. (2004) Fragile-X-associated tremor/ataxia syndrome (FXTAS) in females with the FMR1 premutation. American journal of human genetics 74: 1051-1056.

34. Warren ST, Sherman SL (2001) The fragile X syndrome. In: Scriver CR, Beaudet AL, Valle D, Childs B, Kinzler KW, Vogelstein B (Eds). The Metabolic \& Molecular Bases of Inherited Disease (New York: McGraw-Hill Companies), pp: 1257-1290.

35. Sherman S (2002) Epidemiology. In Fragile X Syndrome: Diagnosis, Treatment and Research, R.J. Hagerman, ed. (Baltimore, MD: The Johns Hopkins University Press), pp: 136-168.

36. Hagerman RJ, Ono MY, Hagerman PJ (2005) Recent advances in fragile $X$ : a model for autism and neurodegeneration. Current opinion in psychiatry 18: 490-496.

37. Greco CM, Hagerman RJ, Tassone F, Chudley AE, Del Bigio MR, et al. (2002) Neuronal intranuclear inclusions in a new cerebellar tremor/ataxia syndrome among fragile $X$ carriers. Brain: $a$ journal of neurology 125: 1760-1771.

38. Tan H, Qurashi A, Poidevin M, Nelson DL, Li H, et al. (2012) Retrotransposon activation contributes to fragile $X$ premutation rCGG-mediated neurodegeneration. Human molecular genetics 21: 57-65.

39. Qurashi A, Li W, Zhou JY, Peng J, Jin P (2011) Nuclear accumulation of stress response mRNAs contributes to the neurodegeneration caused by Fragile $X$ premutation rCGG repeats. PLoS genetics 7: e1002102. 